\title{
Rethinking Physical Activity Promotion During the COVID-19 Pandemic: Focus on a 24-hour Day
}
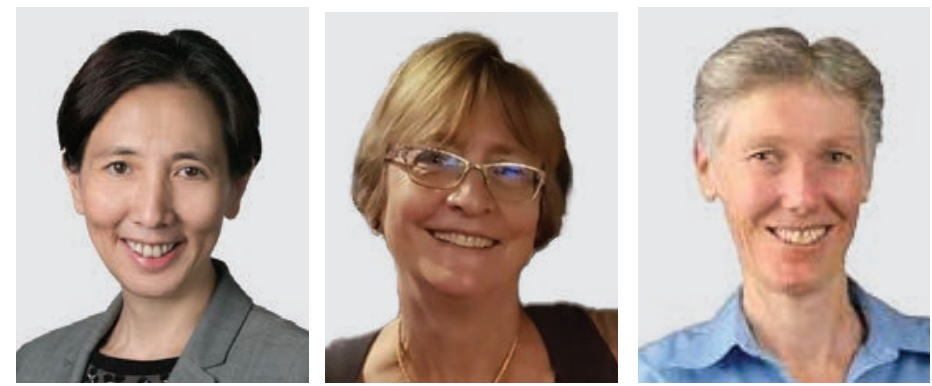

\author{
Linda C. Li ${ }^{1}(\mathbb{D})$ Lynne M. Feehan ${ }^{2}$ (D) and Alison M. Hoens ${ }^{1}$ (D)
}

On March 11, 2020, the World Health Organization (WHO) declared the coronavirus disease 2019 (COVID-19; caused by SARS-CoV-2) a pandemic. By mid-March, all Canadian provinces and territories declared states of emergency, which triggered measures to support the response to the pandemic. ${ }^{1}$ Some of these measures have posed new barriers for people with arthritis to manage their health and daily activities. With the chance of contracting COVID-19 unclear among people using immunosuppressive medications, many avoided regular activities such as grocery shopping and pharmacy visits to reduce exposure in public spaces early in the pandemic. ${ }^{2}$ Gyms and swimming pools were closed, leaving people without access to their regular exercise facilities. Even when facilities reopened as local transmission was getting under control, some people were cautious about resuming indoor and group exercise routines. This unprecedented situation has challenged how health professionals support people with arthritis in staying physically active.

Worldwide, $28.5 \%$ of people were not sufficiently physically active, with nearly $37 \%$ in high-income Western countries. ${ }^{3}$ For more than a decade the main message of physical activity promotion has been centered on achieving concrete goals, namely at least 150 minutes/week of moderate/vigorous physical activity (MVPA), plus strength training at least 2 days a week for adults aged 18 or older. ${ }^{4}$ Further, people aged 65 and over are advised to engage in multicomponent physical activity that includes balance training for fall prevention. These recommendations are echoed in the 2020 WHO guidelines, ${ }^{5}$ in which the phrase "every move counts" was coined to encourage participation in

LCL holds the Harold Robinson/Arthritis Society Chair in Arthritic Diseases, and is supported by the Canada Research Chair Program.

${ }^{I}$ L.C. Li, PT, PhD, A.M. Hoens, PT, MSc, Patient Partner, Department of

Physical Therapy, University of British Columbia, and Arthritis Research Canada; ${ }^{2}$ L.M. Feehan, PT, PhD, Department of Physical Therapy, University of British Columbia, Vancouver, British Columbia, Canada.

Address correspondence to Dr. L.C. Li, Department of Physical Therapy, University of British Columbia, 2177 Wesbrook Mall, Vancouver, BC

V6T 1Z3, Canada.Email: lli@arthritisresearch.ca. any type of physical activity at any duration, and to reduce sedentary time. A recent editorial by Jakiela, et al ${ }^{6}$ also recommends that patients with arthritis start by walking 10 minutes/day during the pandemic and gradually increase duration and speed.

\section{Increase physical activity: an implementation challenge}

Maintaining an active lifestyle is a core component of self-care in people with arthritis, but for some, introducing physical activity in their daily lives is not a simple process. In addition to the common barriers, such as a lack of time and opportunities, people's activities can be hindered by arthritis symptoms and disease activity. ${ }^{7}$ A recent qualitative study revealed the experiences of people with rheumatoid arthritis making extra effort to plan their exercises, especially if they anticipated that an increase in pain and fatigue after activity might affect how much they could do for the rest of the day. ${ }^{8}$ Thus, focusing solely on concrete goals (e.g., 150 minutes of weekly MVPA) without acknowledging the other factors within the context of an individual's daily life is insufficient to move the needle of physical activity promotion.

Guideline implementation can be complex when it involves "things" that people perceive as hard to "do." The concepts related to implementation are eloquently explained by Curran" as "the thing" and "do the thing." "The thing" refers to an intervention or a practice that is in need of support (e.g., achieving 150 minutes of MVPA every week). To help people to "do the thing," an implementation strategy that supports them overcoming barriers is often needed. The development and evaluation of these strategies are the crux of implementation research. Here, we propose that to make physical activity guidelines usable, sensible implementation strategies that take into account individuals' contexts are needed, particularly with the evolving situation of the pandemic.

The focus on achieving 150 minutes/week $v$ s balancing activities in a 24-hour day

We all have 24 hours in our day and what we each do with that time can differ. In a study of 172 people with arthritis who wore 
a physical activity sensor over 7 days, Feehan, et al ${ }^{10}$ identified 4 distinct activity profiles based on the average time they spent each day sleeping, sitting, and walking. The latter includes walking during usual daily activities and brisk walking during higher-intensity activities. In the Balanced Activity profile, people spent an average of 48 minutes/day in higher-intensity walking activities, 4.4 hours/day walking during light activities, 9.4 hours/day in sedentary activities, and 7.4 hours/day sleeping. In contrast, the High Sitters were getting adequate sleep, but sitting more than 13 hours/day. The High Sleepers included people who slept more than 8.5 hours/day. They also spent 2.5 hours/day in light activity, but only 18 minutes/day in higher-intensity walking. Last, the Low Sleepers slept an average of 6.5 hours, and accumulated over 12 hours of sitting each day, similar to the High Sitters. Notably, existing habits related to walking and sitting play a role in how people spent their time.

These findings suggest that people may need different strategies to achieve a healthy balance between physical activity and rest. For example, sleep-related interventions may be needed as a physical activity promotion strategy in people who fit the High Sleeper and Low Sleeper profiles. While the High Sleepers may benefit from shifting some of their sleep time to any level of physical activity, the Low Sleepers may focus on gaining more sleep and walking by reducing seated activities. These examples challenge the current thinking of "the thing" in physical activity promotion.

\section{Rethink physical activity promotion}

By refocusing on balancing activities in a 24-hour day, health professionals and implementation scientists can begin to design strategies that tailor their support for patients according to their capacity, opportunity, and motivation ${ }^{11}$ to engage in physical activity. This effort can be facilitated by the new Canadian 24-hour Movement Guidelines. ${ }^{12}$

Released in October 2020, the Canadian guidelines promote a balance of activity, rest, and sleep as playing an important role for better overall health and quality of life regardless of age and health conditions. ${ }^{12}$ People are encouraged to shift their sedentary time to engage in physical activity at any level whenever they are able, while maintaining a healthy amount of sleep. For people with arthritis, this approach may be more acceptable, especially during the pandemic when their activity patterns may change depending on their local pandemic response plan and personal preferences. It also highlights the importance for health professionals to address sleep and fatigue when codesigning a physical activity intervention with their patients.

Reimagine how to "do the thing"

The success of promoting a balanced activity profile depends partly on how an intervention is delivered. In a systematic review of behavior change techniques, Ma and Martin Ginis found interventions that included feedback on behavior, self-monitoring, instructions on how to perform the behavior, and problem solving with patients tended to have greater effects on physical activity behavior compared to those using other techniques. ${ }^{13}$ These behavior change techniques are defined in the literature, ${ }^{14}$ but the execution may require tailoring, and involves gathering relevant information from patients to inform the design of a personalized plan. To this end, a counseling technique using the Brief Action Planning approach, combined with the use of a fitness tracker to provide feedback on performance, is an example of a promising strategy for improving the delivery process. ${ }^{15,16}$

In addition to physicians, the physiotherapists, nurses, and occupational therapists who work with patients in a variety of settings in the public and private sectors are in an ideal position to provide activity counseling, especially for those with multimorbidity. Training on the Brief Action Planning approach, which used to be offered only in-person, ${ }^{15,16}$ is now available online for rheumatology professionals. The ubiquitous use of self-monitoring devices by the public, advances in virtual care technologies, and an unprecedented increase in the use of telehealth during the pandemic have also generated new opportunities for health professionals to tailor and deliver interventions supporting patients to be active.

It is, however, important to be mindful about inequities that exist in promoting physical activity to "hardly reached populations ${ }^{17}$ based on demographic characteristics, societal factors, and living conditions. We encourage health professionals and researchers to apply the PROGRESS-Plus framework when considering their approach to develop strategies for promoting a balanced activity profile across populations. PROGRESS stands for "place of residence, race/ethnicity, occupation, gender, religion, education, socioeconomic status, and social capital and networks." Age, disability, and sexual orientation were later added to the grid for the PROGRESS-Plus. As people's experiences are shaped by social factors, the PROGRESS-Plus framework offers a useful filter to identify determinants of inequality and inequity, ${ }^{18}$ which can in turn inform an intersectional approach ${ }^{19}$ when designing strategies to support patients during and beyond this pandemic.

Finally, health professionals may consider beginning each physical activity conversation by asking patients how they spend their time on a typical weekday and a typical day in a weekend. This offers an opportunity to gather relevant contextual information to codevelop a realistic 24-hour activity/sleep plan and identify the support required to put it in action.

\section{Conclusion}

Changing behavior is a complex task, and sometimes additional effort is needed to support patients to move more, sit less, and get a healthy amount of sleep. The pandemic has imposed unusual challenges on the self-care activities of people with arthritis. But it also presents a unique opportunity for the rheumatology community to embrace a holistic approach so that we can support patients to integrate more physical activity in their daily lives.

\section{REFERENCES}

1. Vogel L. COVID-19: a timeline of Canada's first-wave response. CMAJ News 2020. [Internet. Accessed February 5, 2021.] Available from: cmajnews.com/2020/06/12/coronavirus-1095847 
2. Michaud K, Wipfler K, Shaw Y, Simon TA, Cornish A, England $\mathrm{BR}$, et al. Experiences of patients with rheumatic diseases in the United States during early days of the COVID-19 pandemic. ACR Open Rheumatol 2020;2:335-43.

3. Guthold R, Stevens GA, Riley LM, Bull FC. Worldwide trends in insufficient physical activity from 2001 to 2016: a pooled analysis of 358 population-based surveys with 1.9 million participants. Lancet Glob Health 2018;6:e1077-86.

4. Tremblay MS, Warburton DE, Janssen I, Paterson DH, Latimer AE, Rhodes RE, et al. New Canadian physical activity guidelines. Appl Physiol Nutr and Metab 2011;36:36-46.

5. Bull FC, Al-Ansari SS, Biddle S, Borodulin K, Buman MP, Cardon G, et al. World Health Organization 2020 guidelines on physical activity and sedentary behaviour. Br J Sports Med 2020;54:1451-62.

6. Jakiela JT, Waugh EJ, White DK. Walk at least 10 minutes a day for adults with knee osteoarthritis: recommendation for minimal activity during the COVID-19 pandemic. J Rheumtol 2021; 48:157-9.

7. Jordan JL, Holden MA, Mason EE, Foster NE. Interventions to improve adherence to exercise for chronic musculoskeletal pain in adults. Cochrane Database Sys Rev 2010;2010:CD005956.

8. Ma J, Collins J, Davidson E, English K, Hoens AM, Tsui K, et al. Strength training for people with rheumatoid arthritis: barriers, facilitators, and tailoring considerations [abstract]. Arthritis Rheumatol 2019;71 Suppl 10.

9. Curran GM. Implementation science made too simple: a teaching tool. Implement Sci Commun 2020;1:27.

10. Feehan LM, Lu N, Xie H, Li LC. Twenty-four hour activity and sleep profiles for adults living with arthritis: habits matter. Arthritis Care Res 2020;72:1678-86.

11. Michie S, van Stralen MM, West R. The behaviour change wheel: a new method for characterising and designing behaviour change interventions. Implement Sci 2011;6:42.

12. Ross R, Chaput JP, Giangregorio LM, Janssen I, Saunders TJ, Kho ME, et al. Canadian 24-hour movement guidelines for adults aged
18-64 years and adults aged 65 years or older: an integration of physical activity, sedentary behaviour, and sleep. Appl Physiol Nutr, and Metab 2020;45:S57-102.

13. Ma JK, Martin Ginis KA. A meta-analysis of physical activity interventions in people with physical disabilities: content, characteristics, and effects on behaviour. Psychol Sport Exerc 2018;37:262-73.

14. Michie S, Richardson M, Johnston M, Abraham C, Francis J, Hardeman W, et al. The behavior change technique taxonomy (v1) of 93 hierarchically clustered techniques: building an international consensus for the reporting of behavior change interventions. Annal Behav Med 2013;46:81-95.

15. Li LC, Sayre EC, Xie H, Falck RS, Best JR, Liu-Ambrose T, et al. Efficacy of a community-based technology-enabled physical activity counseling program for people with knee osteoarthritis: proof-of-concept study. J Med Internet Res 2018;20:e159.

16. Li LC, Feehan LM, Xie H, Lu N, Shaw C, Gromala D, et al. Efficacy of a physical activity counseling program with use of a wearable tracker in people with inflammatory arthritis: a randomized controlled trial. Arthritis Care Res 2020;72:1755-65.

17. BC Centre for Disease Control. BCCDC COVID-19 language guide. Guidelines for inclusive language for written and digital content. [Internet. Accessed February 5, 2021.] Available from: www.bccdc.ca/Health-Info-Site/Documents/Language-guide.pdf

18. Guillemin F, Carruthers E, Li LC. Determinants of MSK health and disability - social determinants of inequities in MSK health. Best Pract Res Clin Rheumatol 2014;28:411-33.

19. Knowledge Translation Program. Intersectionality \& Knowledge Translation (KT). Intersectionality guide. [Internet. Accessed February 5, 2021.] Available from: knowledgetranslation. net/wp-content/uploads/2020/08/Intersectionality_KT_ Guide_20200317_FD.pdf 